\title{
Perilla oil prevents brain damage via suppressing risk factor protein expression in hypercholesterolemic apo E KO mice
}

\author{
J. E. Seong, J. E. Lee, H. E. Hyun, E. H. Lee and Y. O. Song \\ Department of Food Science and Nutrition, Kimchi Research Institute, Pusan National University, Busan 609-735, Korea
}

Hypercholesterolemia might bring on the neurological disorders such as dementia ${ }^{(1)}$. On recent studies, $n$ - 3 fatty acid is known to relieve brain disorders by preventing the oxidative damage on neural cells ${ }^{(2)}$. This study is aimed for demonstrating the beneficial effects of perilla (PE) oil, rich in $n-3$ fatty acid, on neural damage induced by high cholesterol diet (HCD). The diet contains $1.25 \%$ cholesterol, $4 \%$ cocoa butter, $12 \%$ coconut oil and $10 \%$ of various cooking oils. Lard (LD), PE or sunflower (SN) oil are used for a source of $n-9, n-3$ and n-6 fatty acid, respectively. The diet was provided to the apo E KO mice and its wild type, C57BL/6 mice for 10 weeks. The animal protocol used in this study has been reviewed by the Pusan National University-Institutional Animal Care and Use Committee (PNUIACUC) on their ethical procedures and scientific care, and it has been approved (Approval Number PNU-2010-00031). For histological analysis, brain tissue was sectioned followed by hematoxylin-eosin staining and damaged cell were counted under the optical microscopy using Towfigh's method ${ }^{(3)}$. Western blotting is performed for detecting the risk factor protein responsible for the brain oxidative damage. These data were analysed by using ANOVA followed by Duncan's multiple.
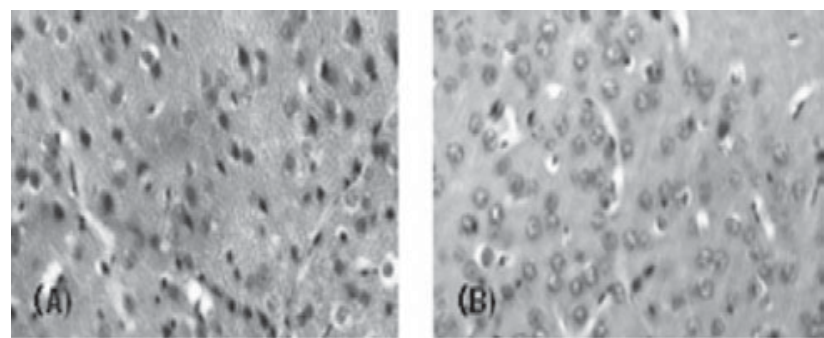

Fig. 1. Photomicrographs of cerebral cortex in the brain. (A): LD group (B): PE group, (Hematoxylin-eosin, X200).

Table 1. Degree of brain cell damage. Data with different letters are significantly different $(P<0.05)$.

\begin{tabular}{llcc}
\hline & & \multicolumn{2}{c}{ Histological damage } \\
\cline { 3 - 4 } Mouse model & Diet & Mean & SD \\
\hline Wild-type & LD & $9.25^{\mathrm{bc}}$ & 2.38 \\
& PE & $6.60^{\mathrm{c}}$ & 1.74 \\
\multirow{2}{*}{ Apo E KO-type } & LD & $14.75^{\mathrm{a}}$ & 2.28 \\
& PE & $9.60^{\mathrm{bc}}$ & 2.24 \\
& SN & $12.40^{\mathrm{ab}}$ & 2.58 \\
\hline
\end{tabular}

Fig. 1. is photomicrographs of cerebral cortex in the brain of wild type mouse fed HCD for 10 weeks. Brain damage was found to be less severe in the group of WPE, WLD, APE, ASN, ALD, in order. Cerebral cortex damage for the APE group was significantly reduced by $29.4 \%$ than ALD group $(P<0.05)$. In our previous study, TC and TG concentration as well as degree of lipid peroxidation in the brain for APE group were the lowest among the experimental groups. These results inform us that the beneficial effects of $n$ - 3 fatty acid under hypercholesterolemic conditions $(P<0.05)$. Over expressed COX-2, iNOS, IkB and NFkB, the risk factors responsible for the neural oxidative damage by HCD were significantly reduced in the APE group $(P<0.05)$. In conclusion, PE oil, rich in $n-3$ fatty acid, seems to have beneficial effects on neural cell damage caused by hypercholesterolemia via suppressing the expression of brain injury risk factor proteins through inhibiting the lipid accumulation in the brain.

This work was supported by the research grant (no. 109130-3) from the Ministry for Food, Agriculture, Forestry and Fisheries, Republic of Korea, which is gratefully appreciated.

1. Refolo LM, Pappolla MA, Malester B et al. (2000) Neurobiol Dis 7, 321-331.

2. Marianna M, Massimilliano P, Luigi J et al. (2007) Prog Neuropsychopharmacol Biol Psychiatry 31, 12-26.

3. Towfighi J, Housman C, Heitjan DF et al. (1994) Acta Neuropathol 87, 598-604. 